\title{
Prevalence and associated factors of erectile dysfunction among men DM patients in Gondar university hospital, Gondar Ethiopia
}

tewodros tesfaye ( $\sim$ tedomarpt@gmail.com )

unversity of gondar

mulugeta bayisa

unversity of gondat

Nebiyu mesfin

unversity of gondar

Research Article

Keywords: erectile dysfunction,DM,prevalence,ethiopia

Posted Date: January 16th, 2020

DOI: https://doi.org/10.21203/rs.2.20496/v1

License: (c) (1) This work is licensed under a Creative Commons Attribution 4.0 International License.

Read Full License 


\section{Abstract}

Back ground- globally it is likely that 387 million people have DM and this number expected to increase to 592 million by the year 2035. WHO estimated the number of cases of diabetes in Ethiopia to be about 800thausadns in 2011 and expected that it would increase to about 1.8 million by the year 2030 . Psychosomatic and sexual dysfunctions are one among different complication that DM patients come across during their disease course. Therefore this study aimed to estimate the prevalence and associated with erectile dysfunction among adult men DM patients in Ethiopia.

Method-An institutional based cross-sectional study design was conducted among male DM patients at Gondar university referral hospital chronic illness clinic. Single population proportion sampling technique was used. 367 were selected proportionally using systematic random sampling technique. Face to face interview method was employed using a structured questionnaire for data collection. Data were analyzed descriptively and through bi-and multivariate logistic regression model.

Result-The prevalence rate is $\mathbf{5 3 . 1 \%}$. The mean age of the respondents was $54.12(\mathrm{SD}+\mathbf{1 6 . 5 1 1 2})$ years age from 21-90 years. Majority of the respondents 158(43.1\%) were Orthodox followers followed by Muslim, $113(30.8 \%)$, Protestant 55(15.0\%) and Catholic 41(11.2\%). the median monthly salary was 2000 Ethiopian Birr. BMI of most respondents was within the range of 18.5-24.9. ED was significantly associated with Age, Duration of DM and Alcohol.

Conclusion- Finding of this study indicated that ED is a major public and self issue with its multi-factorial among male DM. The evidence from this study found that being aged, alcoholic and longer duration of DM was significantly associated with ED.

Key words-Erectile dysfunction, prevalence, male DM patients, Gondar University Referral Hospital.

\section{Background}

Erectile dysfunction (ED) is a common problem of diabetes and vital cause of decreased quality of life in diabetic men(1). ED is a persistent inability to attain and maintain a penile erection sufficient for satisfactory sexual performance and also a wide spread and bothersome problem in middle aged male(2). The international institutes of health $(\mathrm{IIH})$ defines it is the constant inability to preserve a penile erection, sufficiently for satisfactory sexual intercourse(3). ED is a regular inability of the male in achieving or sustaining an erection of adequate rigidity for permitting a satisfactory sexual intercourse, in diabetic men erectile dysfunction usually occurs as a result of micro-vascular changes, neuropathy and endothelial dysfunction(4).

The prevalence varies extensively among studies ranging from $20 \%$ to $70 \%$. Several selective mechanisms could explain these difference among study population as they vary in age, type and severity of diabetes and criteria for diagnosis of ED(1). The prevalence of ED among DM men rises with age, it also seems to 
be allied to the duration of DM, the degree of metabolic control and the presence of other complications(1). The prevalence of ED was $58 \% .94 \%$ of subjects with ED had unsatisfactory control $(\mathrm{HbAl}>7 \%)(P=0.019)$. Increasing age over 45 year, extensively increased the prevalence of $E D(P=0.03)$. $79 \%$ subjects with ED reported decreased self-esteem and $99 \%$ reported that their primary care physician had never discussed this issue with them(5).

A study done in Kuwait the prevalence of ED revealed that the prevalence of erectile dysfunction along with newly diagnosed type 2 diabetes men was 31\% (9). Another cross-sectional study done in India shows the prevalence of erectile dysfunction among DM patients is $58 \%(5)$. Another cross-sectional cohort study done in Tehran, Iran reported that the prevalence of erectile dysfunction among DM patients is $18.8 \%(10)$. Another study done in North India the occurrence of erectile dysfunction among type $2 \mathrm{DM}$ patients is $77.2 \%(4)$. A cross-sectional study conducted among $200 \mathrm{DM}$ patients in Iran the prevalence of erectile dysfunction is $82.5 \%$ ( $\mathrm{N}=200 \mathrm{Men}=100$ and Women=100)(11). Another study done in India to study the prevalence of erectile dysfunction among diabetic men who visited outpatient clinic in Amrita institute of medical science and research center found $58 \%(85 / 147)$ subjects who reported to have history of $\mathrm{ED}(5)$. Another retrospective study done in outpatient diabetic clinic of University of Sprinagarind hospital, Thailand shows $17.5 \%$ (12). Another cross-sectional study done in China the prevalence of erectile dysfunction among type $2 \mathrm{DM}$ patients is $79.1 \%(13)$.

Another study done in Spain the prevalence of erectile dysfunction among DM patients revealed that 31\% (14). Another study done in Italy showed the prevalence of erectile dysfunction among in both type 1 and type 2 DM patients, in type $1(51 \%)$ and in type $2(37 \%)(15)$. A study conducted on the prevalence of erectile dysfunction among type $1 \mathrm{DM}$ patients at outpatient diabetic clinic of Catholic university of Leuven showed $22 \%(16)$. One study done in Italy showed the prevalence of erectile dysfunction among DM patients is $(\mathrm{N}=20) 15 \%(17)$. Another study conducted in London the prevalence of erectile dysfunction among type $1 \mathrm{DM}$ patients is $56 \%$ ( $\mathrm{N}=142$ type $1 \mathrm{DM})(18)$. Another study conducted in Italy the prevalence of erectile dysfunction among type 2 DM patients is $34 \%$ reported frequent erectile dysfunction and $24 \%$ reported irregular problem(19). Another cross-sectional cohort study done in Italy the prevalence of erectile dysfunction among DM patients 35.8\%(1). Another study done in Tel-Aviv Sourasky medical center TEL-Aviv, Israel the prevalence of erectile dysfunction among men DM patients is $57 \%(20)$

A multifactorial association for ED among diabetic male was reported by many studies. A cohort study done in the USA on the threat of diabetics ED showed that smoking, alcohol consumption, had high average of BMI, less physical activity, age and are significantly associated with $\operatorname{ED}(26)$. Another multi centered cross-sectional survey on type 2 DM in urban area of Hong Kong, China the incidence of ED increases in $>60$ years age group is higher than $50 \%(13)$

Another study in Tehran, Iran to find out the risk factor of smoking using five item version of (IIEF-5). The severity of ED allied significantly with the level of experience to smoking so the study reported smoking is a high risk factor of ED among DM patients(27). Another study done in Nigeria study reported that duration of DM is significantly connected with $E D(3)$. 
ED is the most frequent complication and regularly occurring form of a problem in DM patients but it's under recognized and under treated in resource limited countries like Ethiopia. Although DM is being recognized as one of the health problems, its complication like ED in the Ethiopian care setup has not been well documented. Especially there is no evidence in our context regarding diabetes ED. The issue of ED is neglected both from a clinical and research stand point in Ethiopia and especially in our setup. Since the problem is growing and the attention given is less further research that comes with valuable information for decision makers, health care planers, evaluator and medical practitioners is needed.

Hence this is a preliminary study to assess the prevalence of ED among Ethiopian male patients and identify the predictor values of ED. The results of this study will provide more insight about ED in DM male patients of Ethiopia and shall provide information for the physicians, counselors, other decision makers and DM patients as well.

\section{Method}

\section{Study design, period and study area}

An Institutional based cross-sectional study design was conducted in the diabetics unit of chronic illness in UOG referral hospital. The study was conducted in the diabetics unit of chronic illness in UOG referral hospital located $742 \mathrm{~km}$ north from Addis Ababa Ethiopia. It is a regional referral hospital with more than 550 beds and has an outpatient department which serves about 250 patients's every day. The study is conducted from November, 2016 to June, 2017. The source population was all DM patients unit of chronic illness clinic in UOG referral hospital and the study population was male DM patients unit of chronic illness clinic in UOG referral hospital during the period of data collection from November to December 2016. And the total population of Gondar is 333103 and have total of 21 kebeles.

\section{Sample size and sampling.procedures}

The sample size was determined or calculated according to the assumption of single population proportion using the following assumption from the previous study done in Kenya at Kenyatta national hospital, Kenya's main referral and teaching hospital for university of Nairobi $68 \%$ of the people with diabetic men ED using IIEF-5 tool(25).

$Z=95 \%$ confidence interval $=1.96$

$\mathrm{P}=68 \%$ single population proportion

$d=$ marginal error $5 \%$

$$
\mathrm{n}=\underline{\mathrm{Z}} \underline{\underline{2}} \underline{\mathrm{P}}(\underline{1-\mathrm{P}})=1.96^{2} \underline{\mathrm{X} 0.68}(\underline{0.32})=334
$$
$(d)^{2}$
$(0.05)^{2}$ 
Then the final sample size with $10 \%$ non response will be $334+10 \%$ non response $=367$.

Simple random sampling technique was used to collect the data. Every $3^{\text {rd }}$ patient who was coming to the chronic illness clinic are interviewed. Subjects or participants are taken in order to avoid reputation in case of some patients may come for visit more than one times within the period of data collection.

\section{Data collection procedure}

The structured questionnaire was adopted using (IIEF-5) is a well validated questionnaire which is used for the evaluation of sexual dysfunction and it is used to assess ED in diabetic men.

Four male nurses have training for data collection by the investigator, the whole interview part is collected by the trained nurses a structured questionnaire was proposed for interview to collect the sociodemographic information from DM patients following at UOG referral hospital chronic illness clinic, and clinical characteristics was collected from the patient history or record.

\section{Data quality control}

Data collectors were take training for two days. To ensure the consistency of the interview and examination and the acquisition of quality data, random check was carried out for its completeness, accuracy and clarity on a regular base by the principal investigator during the data collection period. The questionnaire have pre-test at one of private clinic for its applicability, simplicity, understandability by the trained data collectors with a very close supervision of the principal investigator.

\section{Data processing and analysis}

Data was going to coded and entered using EPI INFO version 7 and exported to SPSS for windows version 20. Missing values and data clearance was checked. The magnitude and severity in relation to sociodemographic data was analyzed using descriptive analysis. Frequency tables, graphs, percentages were used. Bivariate analysis was conducted primarily to check which variables have association with the dependent variable individually. Variables found to have association with the dependent variable are then entered in to multivariate logistic regression for controlling the possible effect of confounders and finally the variable which has significant association are identified on the basis of odds ratio(OR), with $95 \% \mathrm{Cl}$ and P-value $<0.05$. 


\section{Ethical approval and consent to participation}

Ethical clearance was obtained from the UOG review board. The study purpose and importance is explained to all participants and written consent obtained prior to responding to the interview. They are told that they can refuse to participate. Confidentiality highly maintained at all levels of the study.

\section{Results}

A total of 367 questionnaires were distributed from whom 367 were returned with a response rate of $100 \%$. All of the respondents were male. The mean age of the respondents was $54.12(S D+16.5112)$ years age from 21-90 years. Majority of the respondents 158(43.1\%) were orthodox followers followed by Muslim, $113(30.8 \%)$, protestant $55(15.0 \%)$ and catholic $41(11.2 \%)$. the median monthly salary was 2000 Ethiopian birr. BMI of most respondents was within the range of 18.5-24.9.

Majority 332/367(90.5\%) of the participants was categorized as normal BMI 18.5 and 24.9 .

There was no history of smoking experience from the participants. Around fiftysix percent (56) of participants who responded doing physical exercise 3 times per a week and 64 (17.4\%) doing once a week. $39(10.6 \%)$ of participants who respond have alcohol drinking history. Of 367 participants $44(12 \%)$ have a long duration of $\mathrm{DM}(>30$ years $)$ but majority of the participants $180(44.0 \%)$ have at the level 11-30 years of duration of DM. 72(19.6\%) have 1-5 years of duration and 71(19.4\%) have 6-10 years of duration. Among 367 One year self reported prevalence of erectile dysfunction among 367 male DM participants, those who reported having erectile dysfunction $53.1 \%$ (195) with $95 \% \mathrm{Cl}$. (Respondents $244(66.5 \%$ ) are type II and the rest $123(33.5 \%)$ are type I. 


\begin{tabular}{|c|c|c|c|c|}
\hline \multicolumn{2}{|c|}{ Variables } & \multicolumn{2}{|c|}{ Erectile Dysfunction } & \multirow{3}{*}{$\begin{array}{l}\text { Total } \\
\text { N (\%) }\end{array}$} \\
\hline & & \multirow{2}{*}{$\begin{array}{l}\text { No } \\
\text { N (\%) }\end{array}$} & \multirow{2}{*}{$\begin{array}{l}\text { Yes } \\
N(\%)\end{array}$} & \\
\hline & & & & \\
\hline \multirow[t]{4}{*}{ Age } & $<30$ & $30(17.4)$ & $1(0.5)$ & $31(8.4)$ \\
\hline & $31-44$ & $71(41.3)$ & $19(9.7)$ & $90(24.5)$ \\
\hline & $45-59$ & $53(30.8)$ & $42(21.5)$ & $95(25.9)$ \\
\hline & $>60$ & $18(10.5)$ & $133(68.2)$ & $151(41.1)$ \\
\hline \multicolumn{5}{|c|}{ Religion } \\
\hline \multicolumn{2}{|c|}{ Orthodox } & $68(39.5)$ & $90(46.2)$ & $158(43.1)$ \\
\hline \multicolumn{2}{|c|}{ Muslim } & $47(27.3)$ & $66(33.8)$ & $113(30.8)$ \\
\hline \multicolumn{2}{|c|}{ Protestant } & $31(18.0)$ & $24(12.3)$ & $55(15.0)$ \\
\hline \multicolumn{2}{|c|}{ Catholic } & $26(15.1)$ & $15(7.7)$ & $41(11.2)$ \\
\hline \multicolumn{5}{|c|}{ Marital status } \\
\hline \multicolumn{2}{|c|}{ Married } & $115(66.9)$ & $106(54.4)$ & $221(60.2)$ \\
\hline \multicolumn{2}{|c|}{ Unmarried } & $41(23.8)$ & $3(1.5)$ & $44(12.0)$ \\
\hline \multicolumn{2}{|c|}{ Divorced } & $12(7.0)$ & $30(15.4)$ & $42(11.4)$ \\
\hline \multicolumn{2}{|c|}{ Widowed } & $4(2.3)$ & $56(28.7)$ & $60(16.3)$ \\
\hline \multicolumn{5}{|c|}{ Educational level } \\
\hline \multicolumn{2}{|c|}{ Uneducated } & $24(14.0)$ & $110(56.4)$ & $134(36.5)$ \\
\hline \multicolumn{2}{|c|}{ Primary 1-8 } & $15(8.7)$ & $36(18.5)$ & $51(13.9)$ \\
\hline \multicolumn{2}{|c|}{ Secondary 9-12 } & $62(30.0)$ & $45(23.1)$ & $107(29.2)$ \\
\hline \multicolumn{2}{|c|}{ College and above } & $71(41.3)$ & $4(2.1)$ & $75(20.4)$ \\
\hline \multicolumn{5}{|l|}{ BMI } \\
\hline \multicolumn{2}{|c|}{$<18.5$} & $2(1.2)$ & $0(0.0)$ & $2(0.5)$ \\
\hline \multicolumn{2}{|c|}{$18.5-24.9$} & $151(88.8)$ & $180(90.3)$ & $331(90.7)$ \\
\hline \multicolumn{2}{|c|}{$24.9-29.9$} & $18(10.6)$ & $15(7.7)$ & $33(9.0)$ \\
\hline \multicolumn{2}{|c|}{$>29.9$} & $1(0.6)$ & $0(0.0)$ & $1(0.3)$ \\
\hline \multicolumn{5}{|c|}{ Residence } \\
\hline \multicolumn{2}{|c|}{ Urban } & $135(78.5)$ & $81(41.5)$ & $216(58.9)$ \\
\hline \multicolumn{2}{|c|}{ Rural } & $37(21.5)$ & $114(58.5)$ & $151(41.1)$ \\
\hline
\end{tabular}




$\begin{array}{llll}\text { Occupation } & & & \\ \text { Governmental } & 78(45.3) & 5(2.6) & 83(22.6) \\ \text { Private } & 69(40.1) & 58(29.7) & 127(34.6) \\ \text { Daily laborer } & 5(2.9) & 4(2.1) & 9(2.5) \\ \text { Farmer } & 1(0.6) & 81(41.5) & 82(22.3) \\ \text { Unemployed } & 19(11.0) & 47(24.1) & 66(18.0)\end{array}$

\section{Factors associated with ED}

Resident, Occupational status, Educational status, Duration of DM, Alcohol, physical exercise, marital status and age were significantly associated with ED in the bivarate logistic regression analysis. When the variables were entered in to the multivariate logistic regression analysis, ED was significantly associated with Age, Duration of DM and being alcoholic. 


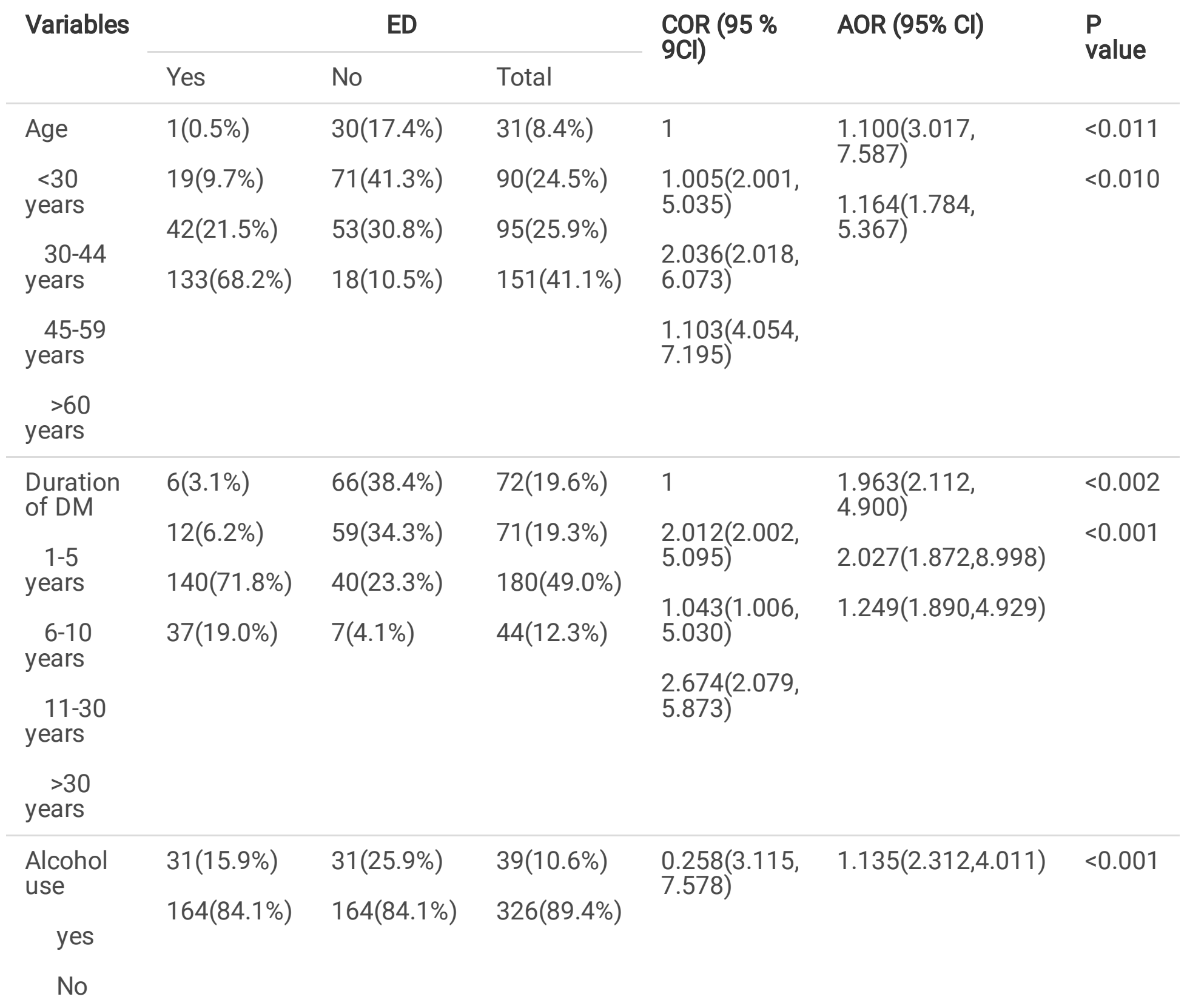

\section{Discussion}

The study estimated the 12 month prevalence of ED and its associated factors among men DM patients at Gondar university hospital. The study found a $53.1 \%$ prevalence of ED among men DM patients. This study which is parallel with studies done in India shows the prevalence of ED among DM is $58 \%(5)$. and a study done in Italy shows the occurrence is $51 \%(15)$. Another study conducted in London the frequency of ED among DM patents is $56 \%(18)$. A study done in Tel-Aviv sourasky medical center Israel the incidence of ED is $57 \%(20)$. Another cross sectional study done in Nigeria shows the prevalence of ED is $57.7 \%(24)$. 
One study in Tanzania whose aim was to determine the prevalence of ED and its associated factors is $55.1 \%(6)$.

However, it is higher than the studies conducted in Kuwait 31\%(9), Theran,Iran 18.8\%(10), Thailand 17.5\% (12), Spain 31\%(14), Italy 22\%(16), Losangles 34\%, Japan 34\%, Malaysia 22\%, Italy $17 \%$ and brazil 15\% (23). The possible reason could be the facility provision difference, the alertness difference for self care and anticipation and in some studies they use only type I DM and duration of DM (1-5 years)(23) and number of study is totally different from us, the possible reason might be due to their high degree of awareness on self care and socioeconomic status are quite different comparing from us.

This study is lower than studies conducted in India 77.2\%(4), Iran 82.5\%(11), China 79.1\%(13). and Kenya $68.8 \%(25)$. The possible reason could be miscellaneous number of participants comparing from us. Other possible reason could be due to different sampling technique and data collection procedure. The study in India and China used postal and phone for data collection but in this study was random sampling technique and direct interview type of data collection procedure was used.

In this study age was significantly associated $(P<0.05)$ with ED. Increasing age increases the odds of developing ED, participants who were in the age greater than 60 years were 0.164 times more likely to report ED as compared to those who were younger than 30 years old ( $\mathrm{AOR}=0.164,95 \% \mathrm{Cl}, 0.41,0.648$ ). While participants who were $45-59$ years were $(A O R=0.100,95 \% \mathrm{Cl}, 0.017,0.587)$. This result is consistent with a study conducted in southern India increase in age to above 45 years was found to significantly increase the occurrence of ED. Age was an important risk factor for ED. Sever ED (IIEF-5) increase from $0.4 \%(20-30$ years) to $0.5 \%(41-50$ years), $1.3 \%$ (51-60years) and to $9.6 \%$ in those who were aged $71-80$ years(4). Another study conducted in Italy increase age was found to increase the odds of ED(15). One of the possible reason for the dissimilarity between older age and younger ones could be that as people get older, weakness of genital and pelvic muscles could happen. A previous study suggests that a likely reason for the higher prevalence of ED among men DM patients is that, as people get older, gradual decline and deterioration of energy happens. Several reasons including changes in the testicles and age related decline in males sex hormones have been adduced for the increasing incidence of ED in older men(3).

In this study, alcohol drinking habit was significantly associated with ED among men DM patients. Participants who had drinking habit were 0.135 times more likely to report ED as compared to non drinkers $(A O R=0.135,95 \% \mathrm{Cl}, 13.075,276,584)$. This result was consistent with the study carried out in Canada alcohol drinking habit was found to increase the odds of $\operatorname{ED}(21)$. The possible reason for the difference between alcohol drinkers and non alcohol drinkers, alcohol lowers the psychological inhibitions that may suppress sexual arousal; alcohol consumption may increase sexual desire, though it also suppresses the associated physiological response. However, excessive alcohol use can cause central sedation, inhibit the spinal reflexes and reduce Lipido, contributing to $\mathrm{ED}(28)$. 
This study showed that duration of DM was significantly associated with ED among DM patients who had long duration of DM were 3.655 times more likely to experience ED when compared to those had short duration of DM history (AOR $=0.04995 \% \mathrm{Cl}, 0.018,0.732$ ). This result consistent with the study conducted in Tanzania confirms that duration of DM were independently allied with $\operatorname{ED}(6)$. The possible reason for the etiology of ED in men DM patients are, sexual active diabetics men have a significant lower cavernosal artery peak blood flow velocity and diameter raise rate than non-diabetics, arterial deficiency due to local microangiopathy, failure of the veins to seal during an erection (veno-occlusive dysfunction) as a result of degenerative changes of the tunica albuginea reported in diabetics can cause ED and penile venous leakage probably produced by autonomic dysfunction of the penile vascular innervations or degeneration of penile smooth muscles either alone or with arterial shortage as a recurrent vascular problem reported in patients with diabetics $\mathrm{ED}(20)$.

\section{Conclusions}

Finding of this study suggested that ED is a major public and self issue with it's multifactorial. This study has led us to conclude that high prevalence of ED among men DM in UOG hospital. The evidence from this study found that being aged, alcoholic and longer duration of DM was significantly associated with ED.

\section{Recommendation}

- In order to reduce prevalence of ED regular exercise is suggested for DM patients.

- In order to reduce the prevalence of ED the patients should get advice not take a drink.

- Findings may be used by hospitals to evaluate the factors of ED among men DM`s and to design the intervention collaborating with physicians and physiotherapists.

- A strong link is recommended to be create between DM patients and physiotherapy department.

- Joint program should be created between Amhara regional health bureau and UOG hospital in collaboration with physiotherapy treatment in ED among men DM patients.

- Further studies, longitudinal ones are required to be carried out for this common problem.

- Efforts must be made to reduce the incidence by dealing with factors responsible for its development.

\section{References}

1. E rectile Dysfunction in Diabetic Subjects in Italy. 1998;21(11).

2. Mohr B, Feldman HA, Ph D, Johannes CB, Ph D, Derby CA, et al. Erectile Dysfunction and Coronary Risk Factors: Prospective Results from the Massachusetts Male Aging Study Erectile Dysfunction and Coronary Risk Factors: Prospective Results from the Massachusetts Male Aging Study. 2000; (October 2016). 
3. Au I, Abasiubong F, la U, Sb U, Bc U. Prevalence and risk factors of erectile dysfunction in Niger delta region , Nigeria. 2012;12(2).

4. Goyal A, Singh P, Ahuja A. Prevalence and Severity of Erectile Dysfunction as Assessed by IIEF-5 in North Indian Type 2 Diabetic Males and Its Correlation with Variables. 2013;7(12):2936-8.

5. Kumar K V, Radhakrishnan AP, Nair V, Kumar H. Erectile dysfunction in diabetic men. 2004;24:23-6.

6. Access 0 . Prevalence of erectile dysfunction and associated factors among diabetic men attending diabetic clinic at Muhimbili National Hospital in Dar-es-Salaam, Tanzania. 2014;8688:1-8.

7. Impact P, Us C, Make TO. Consequences of Erectile Dysfunction. 2016;559:1-5.

8. Relate N. Sex common problems. 2016;9-12.

9. Al-hunayan A, Al-mutar M, Kehinde EO, Thalib L, Al-ghorory M. The prevalence and predictors of erectile dysfunction in men with newly diagnosed with type 2 diabetes mellitus. 2006;130-4.

10. Panel HC. Prevalence and risk factors for erectile dysfunction in a population-based study in Iran. 2003;(November 2002):246-52.

11. Ziaei-rad M, Vahdaninia M, Montazeri A. Sexual dysfunctions in patients with diabetes: a study from Iran. 2010;1-8.

12. Eamudomkarn N, Khampitak K, Sothornwit J, Phunikhom K, Kunatippapong N, Khampitak T, et al. Prevalence and Risk Factors of Erectile Dysfunction in Patient Visiting Andropause Clinic, Srinagarind Hospital , Thailand. 2015;23(1):59-66.

13. Lo WH, Fu SN, King C, Wong H, Chen ES. Prevalence, correlates, attitude and treatment seeking of erectile dysfunction among type 2 diabetic Chinese men attending primary care outpatient clinics. 2014;(December 2013):755-60.

14. Martin-morales A. Prevalence and independent risk factors for erectile dysfunction in Spain: Results of the Epidemiologia de la Disfuncion Erectil Masculina study PREVALENCE AND INDEPENDENT RISK FACTORS FOR ERECTILE DYSFUNCTION IN SPAIN: RESULTS OF THE EPIDEMIOLOGIA DE L. 2016;(October).

15. Fedele D, Bortolotti A, Coscelli C, Santeusanio F, Chatenoud L, Colli E, et al. Erectile dysfunction in Type 1 and Type 2 diabetics in Italy. 2000;19(September 1996):524-31.

16. Prevalence and Predictors of Sexual Dysfunction in Patients With Type 1. 2003;26(2).

17. More Severe Erectile Dysfunction and Worse Quality of Life Than the General Population of Impotent Patients? 2003;26(4):1093-9.

18. Jamieson F, Chalmers J, Duncan C, Prescott RJ, Campbell IANW. Erectile dysfunction in type 1 diabetic males. 44(0):5-7.

19. labetes TYPED, Tudy QUEDS. Erectile Dysfunction and Quality of Life in Type 2 Diabetic Patients. 2002;25(2).

20. Epidemiology C, Aviv T. Prevalence and Risk Factors for Erectile Dysfunction in Men with Diabetes, Hypertension, or Both Diseases: A Community Survey among 1,412 Israeli Men. 2003;30:25-30.

21. Disease V. The Prevalence of Erectile Dysfunction in the Primary Care Setting. 2006;166. 
22. Penson DF, Wessells H, Cleary P, Rutledge BN. NIH Public Access. 2010;6(7):1969-78.

23. Nicolosi A, Moreira ED, Shirai M, Ismail M, Mohd BIN, Glasser DB. COUNTRIES: CROSS-NATIONAL STUDY OF THE ERECTILE DYSFUNCTION. 1998;4295(October 1997):201-6.

24. Unadike BC, Eregie A, Ohwovoriole AE. Original Article Prevalence and types of sexual dysfunction among males with diabetes in Nigeria. 2004;18-20.

25. Likata GMU, Kuria MW, Owiti FR, Likata GMU, Kuria MW, Olando Y. Sexual Dysfunction among Patients with Diabetes Mellitus By. Greener J Med Sci. 2012;2(6):138-45.

26. Acon COGB. Association of Type and Duration of Diabetes With Erectile Dysfunction in a. 2002;

27. Pourmand G, Alidaee MR, Rasuli S, Maleki A, Mehrsai A. Do cigarette smokers with erectile dysfunction benefit from stopping?: a prospective study. 2004;

28. Furukawa S, Sakai T, Niiya T, Miyaoka H, Miyake T, Yamamoto S, et al. Alcohol consumption and prevalence of erectile dysfunction in Japanese patients with type 2 diabetes mellitus: Baseline data from the Dogo Study. Alcohol [Internet]. Elsevier Inc; 2016;55(August):17-22. Available from: http://dx.doi.org/10.1016/j.alcohol.2016.07.006 